\title{
Comparison of simulated and observed large-scale, field-aligned current structures
}

\author{
P. Nenovski \\ Geophysical Institute, Bulgarian Academy of Sciences, 1113 Sofia, Bulgaria
}

Received: 22 January 2007 - Revised: 23 October 2007 - Accepted: 12 November 2007 - Published: 26 February 2008

\begin{abstract}
Recently, a model of large-scale, field-aligned current (FAC) structures, based on zero-frequency MHD surface wave (SW) modes that can emerge from the solar windEarth's magnetosphere interaction, has been proposed. The FAC polarity and intensity distribution are quantified as a function of the solar wind parameters and the interplanetary magnetic field (IMF) magnitude that enter as input parameters. Besides, there are input parameters intrinsic to the Earth's magnetosphere - the size of the polar cap and the boundary regions and their plasma density variations. Influence of the IMF $B_{y}$ component on the FAC structure is examined here. Depending on the IMF $B_{y}$ magnitude, the predicted six-cell FAC structure tends to evolve in a spirallike fashion. This large-scale FAC model is compared with experimental evidences and empirical FAC models based on DE-2 satellite data and high-precision Oersted and Magsat satellite magnetometer data. Among the various achievements of these long-term satellite measurements, an observation/discovery of a ground-based state of FACs which includes a pair of large-scale FACs in the polar cap under both positive and negative IMF $B_{z}$ has been pointed out. The FAC pattern is qualitatively and quantitatively consistent with experimental data for both polar cap FAC and Region 1 and Region 2 FAC systems.
\end{abstract}

Keywords. Magnetospheric physics (Solar-windmagnetosphere interactions; MHD waves and instabilities) Ionosphrer (Electric fields and currents)

\section{Introduction}

Large-scale, field-aligned currents (FACs), also known as Birkeland currents (Potemra, 1985), represent a substantial part of the electric current systems that support the Earth's

Correspondence to: P. Nenovski

(nenovski@geophys.bas.bg) magnetosphere structure during its dynamic interaction with the solar wind variations. Large-scale FACs permeate the Earth's magnetosphere along magnetic field lines toward the ionosphere. At ionosphere heights $(\sim 100-120 \mathrm{~km})$ largescale magnetospheric FACs power ionospheric current systems, like DP2, DP1, DPY, etc. FACs and associated particle precipitations are responsible for heating, excitation and ionization processes in the thermosphere/ionosphere system and therefore, affect our environment. The ionospheric current systems on its own induce electric currents and electric fields in the Earth's subsurface, known as geomagnetically induced currents (GIC) system.

Historically, based on TRIAD magnetometric data, two belts of upward and downward FACs in the polar regions have been statistically discovered and referred to as Region 1 (inner, higher latitude belt) and Region 2 FAC (outer, lower latitude belt) (Iijima and Potemra, 1976a). Another FAC that lies poleward of Region 1 has been observed and termed Cusp or Region $O$ current (Iijima and Potemra, 1976b). When the interplanetary magnetic field (IMF) component $B_{z}$ is positive, i.e. northward, a pair of FACs of inversed polarity poleward of the Region 1 (i.e. in the polar cap region) has been found (Iijima et al., 1984; Zanetti et al., 1984; Stauning, 2002). The latter is referred to as Northward Bz (NBZ) FAC systems. The NBZ FAC system has initially been observed in the southern polar cap region under northward interplanetary magnetic field (IMF) conditions. A sequence of largescale FAC structures of inverse polarity, both in the polar cap and in the auroral region, has been revealed (Akasofu et al., 1980). A three-sheet FAC system that includes cusp (or Region 0), Region 1 and Region 2 currents on the dayside may exist simultaneously (Erlandson et al., 1988). These largescale FAC systems have typical intensities of $\sim 1 \mu \mathrm{A} / \mathrm{m}^{2}$ at ionospheric heights.

More progress with the FAC mapping has been attained with inversions of ground-based magnetograms: IMF control of the high-latitude electric field and associated FAC

Published by Copernicus Publications on behalf of the European Geosciences Union. 
structures has been determined by Greenland magnetometric data (Friis-Christensen et al., 1985); the ionospheric convection and associated FACs in the dayside cusp has been characterized in detail (Lu et al., 1995).

Recently, high-quality magnetic field measurements on board the Magsat, Oersted and CHAMP satellites have provided the unique possibility for studying FAC structures. Various aspects of the large-scale FACs, as well as their dynamics with respect to various geophysical, seasonal and local time (LT) conditions have been considered (Papitashvili et al., 2001; Christiansen et al., 2002; Papitashvili et al., 2002). It has been found that Region 1 and Region 2 FAC systems persist through southward and northward IMF, as well as under IMF $\sim 0$; that both the northern and southern polar caps are filled with FACs even during a slightly southward IMF (Papitashvili et al., 2001). Thus, the latest satellite observations (Oersted and CHAMP) have revealed overall six distinct regions of large-scale FACs, both under northward and southward IMF conditions. A pair of largescale FACs of inverse polarity has been identified inside the polar cap and two pairs of large-scale FACs associated with Region 1 and Region 2 exist equatorwardly of the polar cap FACs. Generally, satellite observations have revealed that the magnetospheric FACs display highly complex and variable structures, where the current densities may vary from a few to several hundred $\mu \mathrm{A} / \mathrm{m}^{2}$ (Stasievicz and Potemra, 1998). Region 1 and Region 2 systems are sometimes distorted and distinct multiple FACs of middle size can be found (Ohtani et al., 1994). Thin FAC sheets of mixed up- and downward currents, up to several hundreds of $\mu \mathrm{A} / \mathrm{m}^{2}$ embedded in largescale FAC systems of only up to a few $\mu \mathrm{A} / \mathrm{m}^{2}$, have often been observed, as well (Stauning et al., 2003).

The following generation mechanisms of the Region 1 and 2 FAC systems are conventionally accepted. Solar windmagnetosphere interaction produces tangential drags at the flanks of the magnetosphere. A tailward motion in the lowlatitude boundary layer (LLBL) is therefore induced. Pressure balance requirements produce internal reverse/return plasma flow in the outer magnetosphere beneath the LLBL. At this interface there is a divergence of the electric field (charge separation) and Region 1 FAC emerges. Region 1 is mapped along magnetic field lines to the conducting polar ionosphere where they are closed (through ionospheric currents). Region 2 FAC emerges at the region where gradient and curvature drift separate charges, thus producing Ring current (RC) and a shielding electric field. Divergence of this shielding electric field at dawn requires the upward $R e$ gion 2 FAC $\mathrm{t}$ be completed by the downward Region 2 FAC at dusk. As for the the polar cap FACs system, Papitashvili et al. (2001) have hypothesized that quasi-viscous interaction of the solar wind with the magnetospheric lobes may cause a sunward convection of the lobes' field lines, mapping largescale FACs of the NBZ-type down to the near pole area.

All the FAC structures, their intensities and variations are closely connected with the solar wind-magnetosphere interaction mechanisms, both viscous and reconnection-like. It has been concluded that out of the evening-midnight sector (18:00-00:00 LT), the Iijima and Potemra's Region 1 and 2 FAC distribution is not affected by reconnection processes (Ogino et al., 1989). Reconnection processes that occur at the magnetopause (especially around the cusp regions) and in the nightside magnetosphere, are considered responsible for the FAC structures of large and medium size around the cusp (Russel and Elphic, 1978, 1979) and in the 18:00-00:00 LT sector, respectively.

Based on Dynamics Explorer 2 measurements, an empirical model of FAC, which maps FACs above the polar ionosphere as a function of the IMF, solar wind velocity, solar wind density and dipole tilt angle has been completed by Weimer (2001a and b). This model reveals conventional Region 1 and Region 2 FAC belts under southward-directed IMF, and a clear NBZ current system surrounded by the Region 1 and Region 2 currents (Weimer, 2001b). In the case of the NBZ current system, a pair of "reversed" convection cells is embedded in a pair of "normal" convection cells at lower latitudes, i.e. the electric potential pattern has a fourcell configuration.

The problems of FAC system modelling are closely connected with the plasma convection patterns in the polar regions, i.e. with the ionospheric electrodynamics. In 1994 Papitashvili et al. (2002) have introduced a linear approach for modeling of large-scale FACs and ionospheric dynamics. This linear approach consists of four basic elements: i) an IMF-independent, two-cell background convection, ii) a lobe convection controlled by the IMF azimuthal component $B_{y}$; and iii) a near-pole, two-cell "reverse" convection caused by a positive IMF $B_{z}$ component, or iv) a merging two-cell convection controlled by a negative IMF $B_{z}$ component. Lately, Papitashvili and Rich (2002) and Papitashvili et al (2002) have shown basic patterns of ionospheric convection and FAC systems, respectively.

Although the ionospheric convection patterns and FAC structures have been, in principle, modelled, all existing relationships between the FAC intensity and distribution and the solar wind and IMF parameters have empirical character. A "geo-effective" solar wind electric field, $E_{\text {eff }}=V_{S W} B_{T} \sin ^{2}(\Theta / 2)$ (where $V_{S W}$ is the solar wind velocity, $B_{T}$ is the transverse IMF and $\Theta$ is the tilt angle defined in the geocentric solar magnetospheric (GSM) coordinates) has been introduced as a quantitative measure of the solar windmagnetosphere interaction and the polar cap potential (Kan and Lee, 1979; Boyle et al., 1997). A viscous mechanism has been suggested for the generation of the polar cap FAC system, its site of generation however is not specified. The underlying physics of the FAC, their intensity and distribution and the associated plasma convection processes in and between adjacent magnetospheric structures through which FACs flow, need therefore further study, in order to be compared with experimental evidences. 
Another problem is the multiple FAC sheet generation in the polar ionospheres. Multiple FAC sheets of medium scales have often been observed by satellites in the nightside polar ionosphere (e.g. Ohtani et al., 1994). Convective plasma vortices of smaller scale have to be associated with these multiple FACs, as well. Indeed, intense variations in the convective electric field magnitude of shorter time scales, measured by microsatellite Astrid-2, have been observed (Eriksson et al., 2002). Their peak-to-peak amplitude often exceeds that of the large-scale (steady) electric field trend. Multiple intense electric field structures are immersed on large-scale convection field structures.

Existing large-scale FAC and plasma convection models cannot simulate such intense variations. Indeed, a wave generation mechanism of multiple FAC structures has also been suggested (Ohtani et al., 1994). There is another possibility. FACs and electric field structures of smaller scales can be interpreted as spatial structures rather than temporal ones. Such an interpretation needs to be grounded by a physical model.

The objectives of the present paper are: (i) to suggest a physical mechanism of existence of the basic, six-region FAC, (ii) to give a model of such a large-scale FAC structure and its quantitative dependence on the solar wind parameters, (iii) to derive a quantitative model of the generation of multiple FAC non-wave (spatial) structures and (iv) to compare FAC characteristics with some well-known models of FAC distribution built on the basis of data assimilation (Weimer, 2001a, b; Papitashvili et al., 2001, 2002).

\section{The surface wave (SW) FAC model}

An existence of a large-scale FAC system of zero-frequency surface modes, supported by the solar wind-Earth's magnetosphere interaction, has been theoretically considered in recent papers by Nenovski (1996, 2003). It is well known that surface wave (SW) magnetohydrodynamic (MHD) modes can emerge at the magnetosphere boundaries. According to theory the SW field is localized at the boundaries (e.g. lowlatitude boundary layer (LLBL), mantle, plasma sheet, etc.). It is hardly, however, is known that SW modes can also support FACs that propagate along the interfaces of zero size (surface FACs), between adjacent regions of different plasma structures. SW modes can thus support boundary FACs along the boundaries of finite size that emerge between the solar wind and the outer magnetosphere (Nenovski, 1996).

The solar wind-magnetosphere interaction model is assumed to be nonlinear; this means that the derived large-scale FAC structures and densities are quantitatively related to solar wind and interplanetary magnetic field (IMF) parameters.

The geometry of the solar wind-magnetosphere interaction is of key importance for large-scale, zero-frequency SW modes generation and the boundary FACs supported by them. The magnetic field lines of the high-latitude Earth's

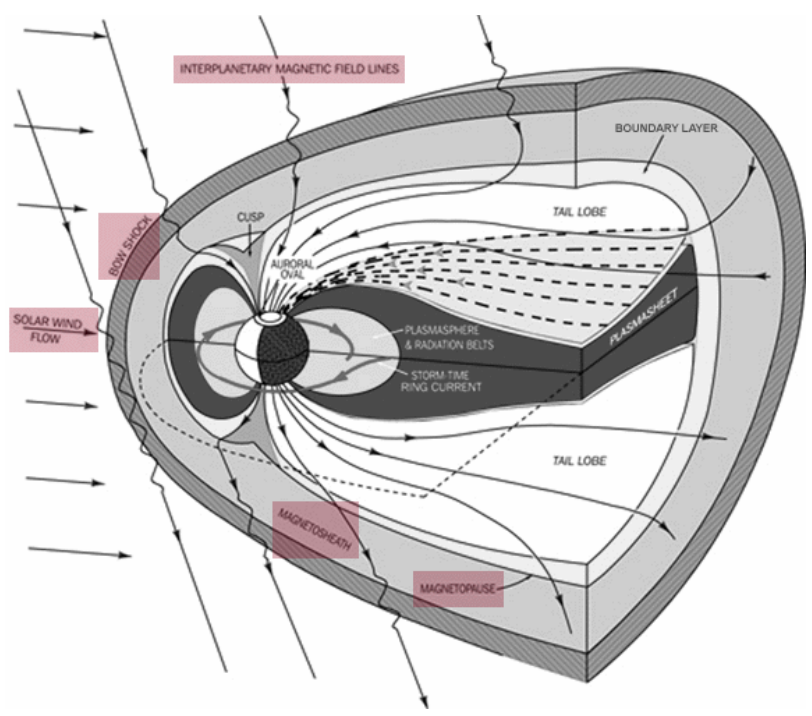

Fig. 1. 3-D picture of the Earth's magnetosphere (adapted from http: //pluto.space.swri.edu/IMAGE/glossary/magnetosphere.html). The plasma sheet and the tail lobe regions and cusps are distorted by the solar wind flow. The solar wind (exterior region) is outside the magnetopause. The boundary region is completed by the boundary layers, cusp, and the plasma sheet. The inner region contains tail lobe field lines that permeate the polar cap region.

magnetosphere regions widen like a funnel at radial distances $1 R_{e} \leq r \leq 10 R_{e}$ ( $R_{e}$ is the Earth's radius). At greater distances, e.g. at $10 R_{e} \leq r \leq 15 R_{e}$, The solar wind blows the two (northern and southern) high-latitude magnetosphere regions, flows around them and partly penetrates (see Fig. 1). The solar wind pressure becomes comparable to the Earth's magnetic field pressure. The latter then cannot oppose the solar wind pressure, and the Earth's magnetic field lines begin to be distorted. The solar wind direction, $\boldsymbol{V}_{0}$, however, is still nearly perpendicular mainly to the (dayside) Earth's magnetic fluxes. At greater distances, e.g. at $r>20 R_{e}$, the Earth's magnetic field lines are practically aligned to the solar wind velocity $\boldsymbol{V}_{s w}$. At distances where the solar wind velocity and the Earth's magnetic field are practically parallel, the well-known Kelvin-Helmholtz instability and SW modes are easily excited. Another SW mode excitation mechanism exists, provided that the solar wind flow direction is nearly perpendicular to the Earth's magnetic fluxes. This happens at distances where the angle between the solar wind velocity and the magnetic field lines of the outer magnetosphere is close to $\pi / 2$, i.e. at distances $r \leq 15 R_{e}$. The latter mechanism of SW mode generation has been examined and modeled (Nenovski, 1996, 2003).

The high-latitude near-Earth magnetospheric regions (i.e. between distances $10 R_{e}<r<15 R_{e}$ ) are assumed to be a flux tube of radius $\rho_{1}$ (a cylindrical geometry). The existence of a unified boundary region of free size $\rho_{1}-\rho_{0}$, where $\rho_{0}$ and $\rho_{1}$ are the inner and outer radii, has been suggested. 
This boundary region is characterized by plasma parameters, whose content is different from those of the solar wind (defined as an open region of radius $\rho>\rho_{1}$ ) and of the inner region of radius $\rho<\rho_{0}$ (that is the polar cap region). Thus, the magnetic flux tube (that interacts with the solar wind flow) consists, on the one hand, of tail lobe field lines (the inner region) of radius $\rho<\rho_{0}$, and on the other hand, of mantle, lowlatitude boundary layer (LLBL), and the plasma sheet boundary regions. The latter are integrated in a unified boundary region lying between $\rho_{0}$ and $\rho_{1}$. The SW mode field distribution is expected to be localized at and around this boundary region (Nenovski, 2003). According to the magnetic flux conservation principle, all model results (e.g. FAC intensity, SW mode magnitude, etc.) obtained at distance $r \approx 10-15 R_{e}$ can be easily mapped to ionospheric heights. Roughly speaking, the situation of SW mode excitation by the solar wind at distance $r \geq 10 R_{e}$ and their propagation properties (along the magnetic field lines to the Earth, i.e. at $r<10 R_{e}$ ) might be considered to be analogous to a propagation of electromagnetic signal along a transmission line. The point where this signal is forced is referred to as the source point. Conditions for generation of the SW modes of largest wavelengths, i.e. those of nearly zero-frequency domain, have been considered (Nenovski, 1996). It is worth noting that even in zerofrequency limit, SW modes still propagate along the Earth's magnetic field lines with the Alfvén speed $V_{A}$.

Equations that govern the SW mode FAC structure propagation are the MHD system and the ionospheric potential equation. The ionosphere is considered as a two-dimensional shell (Kelley, 1989; Raeder, 2003; Moretto et al., 2006):

$\nabla \cdot(\Sigma \cdot \nabla \Phi)=-j_{\|}\left(r_{I}\right) \sin (I)$,

where $\Phi$ is the electric potential, $\Sigma$ is the ionospheric conductance tensor, $I$ is the Earth's magnetic field inclination angle, $j_{\|}\left(r_{I}\right)$ is the field-aligned current (FAC) intensity calculated at ionospheric heights. The FAC intensity at ionospheric heights $j_{\|}\left(r_{I}\right)$ is determined by transforming the FAC intensity and distribution $j_{\|}\left(r_{F A C}\right)$ obtained from the ideal MHD equations applied at source heights $r_{F A C} \approx 10 R_{e}$.

The geometry of the SW mode FAC system model is, however, tied to the Earth's magnetic dipole. Due to the Earth's magnetic dipole rotation, the modeled "flux tube" axis creates a variable angle with an eclipse and solar wind velocity $\boldsymbol{V}_{0}$. The solar wind and interplanetary magnetic field (IMF) parameters thus have to be transformed to the Earth-centred (geocentric) solar magnetospheric coordinate system (GSM) (Russell, 1971). The latter has its $\mathrm{x}$-axis from the Earth to the Sun. The y-axis is defined to be perpendicular to the Earth's magnetic dipole, so that the $x-z$ plane contains the dipole axis. The positive $z$-axis is chosen to be in the same sense as the northern magnetic pole. The northern magnetic pole that coincides with the flux tube orientation creates an angle $\Theta$ (tilt angle) with the $Z$ axis. The IMF has two components, $B_{0 z}$ and $B_{0 \perp}$, oriented along the $Z$ axis and perpendicular to it. It is worth noting that components $B_{0 z}$ and $B_{0 \perp}$

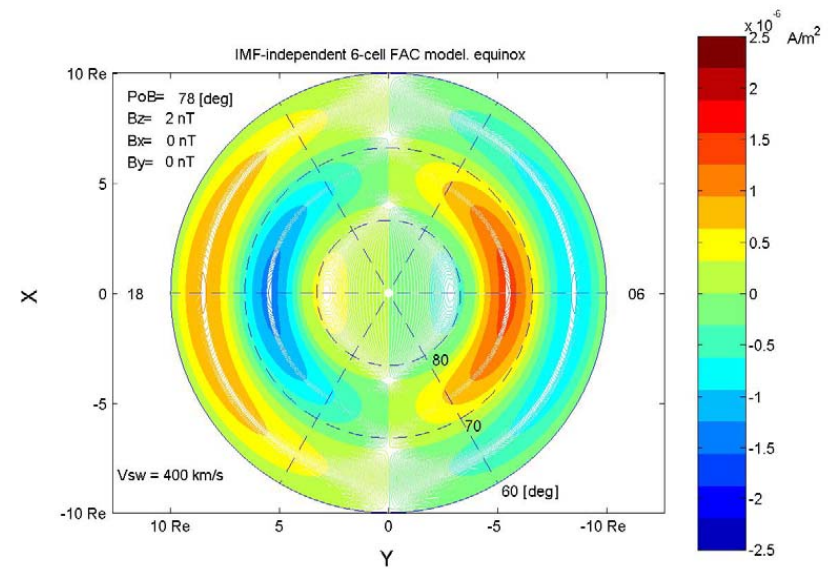

Fig. 2. This figure depicts a six-cell, large-scale FAC system (a ground state) under equinox conditions where the IMF perpendicular components, $B_{x}$ and $B_{y}$, are taken to be equal to zero. The polar cap boundary $(\mathrm{PoB})$ is taken at $78^{\circ}$ magnetic degrees and corresponds to an IMF equal to $2 \mathrm{nT}$. Note that this six-cell FAC system can also exist under zero IMF $B_{z}$ conditions. The FAC intensity is evaluated at ionospheric heights (given in polar coordinate system), taking into account the magnetic flux conservation principle. The source region is assumed at a radial distance $r$ equal to $10 R e$ (shown by X0Y coordinates). Red color and positive FAC intensity values correspond to inward FAC flowing toward the ionosphere, blue color and negative FAC values - to outward FAC flowing away from the ionosphere. The FAC intensity distribution possesses a maximum around $1 \mu \mathrm{A} / \mathrm{m}^{2}$

differ from conventional IMF $B_{x}, B_{y}, B_{z}$ components in the Geocentric Solar Ecliptic (GSE) coordinate system.

In the zero-frequency approximation a stream function (potential) $\varphi$ and vector magnetic field potential $\boldsymbol{A}$ of the SW modes are well introduced. Then

$v=\operatorname{rot} \varphi+\boldsymbol{V}_{0}$ and $\boldsymbol{b}=\operatorname{rot} \boldsymbol{A}$,

where $\boldsymbol{V}_{0} \equiv \boldsymbol{V}_{s w}(\Theta)$ is the solar wind flow mapped on the chosen geocentric GSM coordinate system. It depends on $\Theta$. The solar wind speed, $\boldsymbol{V}_{s w}$ and the IMF components are assumed to be homogeneous.

The following basic equation of the velocity stream function $\varphi$ has been derived (Nenovski, 1996):

$$
\begin{aligned}
& {\left[\varphi-V_{0} y, \Delta \varphi\right]=R^{-2}\left[D\left(\varphi-V_{0} y\right), P\right]+} \\
& R^{-1}\left[M\left(\varphi-V_{0} y\right), \Delta M\left(\varphi-V_{0} y\right)\right]
\end{aligned}
$$

where $R$ and $P$ are plasma density and pressure, respectively. Magnetic field vector potential $\boldsymbol{A}$ and pressure $P$ are expressed by

$A=m\left(\varphi-V_{0} y\right)+\gamma, P=d\left(\varphi-V_{0} y\right)+\delta$,

where all coefficients entering (3) have to be derived by applying boundary conditions at infinity and interfaces between adjacent structures. The basic Eq. (2) is resolved for 
the following boundary conditions: i) the continuity of the potentials (velocity and magnetic field) through interfaces $\rho=\rho_{0,1}$; ii) a constancy of the potentials along circumferences $\rho=\rho_{0,1}$; and iii) the finiteness of the SW mode energy at infinity, $\rho \rightarrow \infty$. The latter means that we have to meet the condition for zero magnitude of SW mode potentials at infinite. Vector magnetic field potential $A$ is related to the SW velocity stream function $\varphi$ (3). Coefficient $m$ (that relates the magnetic field and velocity potentials) is thus equal to $B_{0} / V_{A}$ (Walén relation), where $B_{0}$ is the Earth's magnetic field magnitude, $V_{A}$ is the Alfvén velocity. The FACs distribution and propagation dynamics are described by relation

$j_{\|}=-\mu_{0}^{-1} \Delta A$.

The model yields a FAC intensity and distribution that depends directly on the solar wind and magnetosphere parameters. In general, the SW mode magnetic field and the FAC intensity in all FAC cells are controlled by the density/pressure gradient scale $\lambda_{e}$ at the Earth's magnetopause (Nenovski, 2003), defined by:

$\left.\lambda_{e} \equiv\left(R_{0}^{-1} d R_{0} / d x\right)^{-1}=P_{0}^{-1} d P_{0} / d x\right)^{-1}$.

Gradient $\nabla P_{0}$ is equal to $1 / 2 R_{s w} V_{0 E}^{2} / \rho_{1}$, where $R_{s w}$ and $V_{0 E}$ are the solar wind density and the magnitude of the Earth's orbital velocity, respectively. The FAC intensity distribution, being connected to the plasma pressure gradient scale $\lambda_{e}$, will be controlled by solar wind velocity $V_{0}$, the IMF magnitude and orientation, As expected, the large-scale FAC intensity dynamics depends also on internal parameters: the Earth's magnetic field magnitude (at the magnetopause), $B_{0}$; the plasma density distribution, $R$ (entering through the Alfvén velocity $V_{A}$ ); the tilt angle, $\Theta$ and characteristic scale, $\lambda_{F A C}$, that is inversely proportional to the boundary region cross size $\rho_{1}-\rho_{0}$, i.e.

$\lambda_{\mathrm{FAC}} \propto\left(\rho_{1}-\rho_{0}\right)^{-1}$.

In previous analyses (Nenovski, 1996, 2003), the IMF magnitude and orientation have been coupled with the solar wind velocity magnitude $V_{0}$. The large-scale FAC intensity is thus a function of the IMF $B_{x}$ component, i.e. the previous FAC model concerns an IMF-dependent FAC solutions. In the present study, all the IMF components, $B_{x}, B_{y}$ and $B_{z}$, are taken into account, and the IMF and solar wind velocity potentials are decoupled. Both IMF $B_{x}$ and $B_{y}$ effects are treated here and represent a particular solution of the SW mode FAC model. The large-scale FAC structure is again determined by general dispersion equations derived for arbitrary IMF $B_{x}$ and $B_{z}$ and zero IMF $B_{y}$. Due to the Earth's magnetic field dipole axis orientation, the projection of the IMF $B_{z}$ component onto the equatorial plane of the Earth's magnetic field will additionally change the FAC current distribution.

Without quoting all mathematical procedures, the following dispersion equation that determines all the structural parameters of nonlinear (NL) SW modes and the associated
FACs system under assumed geometry is derived:

$$
\begin{aligned}
& k^{2} r_{1}^{2}\left[-Y_{1}\left(k \rho_{1}\right) J_{1}\left(k \rho_{0}\right)+Y_{1}\left(k \rho_{0}\right) J_{1}\left(k \rho_{1}\right)\right] K_{2}\left(q_{e} \rho_{1}\right) / \\
& K_{1}\left(q_{e} \rho_{1}\right)+q_{e} k \rho_{1}^{2}\left[Y_{1}\left(k \rho_{0}\right) J_{2}\left(k \rho_{1}\right)-Y_{2}\left(k \rho_{1}\right) J_{1}\left(k \rho_{0}\right)\right] \\
& =2 q_{e} \rho_{0} / \pi,
\end{aligned}
$$

$$
\begin{aligned}
& q_{e} \rho_{1}\left[-Y_{2}\left(k \rho_{0}\right) J_{2}\left(k \rho_{1}\right)+Y_{2}\left(k \rho_{1}\right) J_{2}\left(k \rho_{0}\right)\right] \\
& +k \rho_{1}\left[Y_{1}\left(k \rho_{1}\right) J_{2}\left(k \rho_{0}\right)-Y_{2}\left(k \rho_{0}\right) J_{1}\left(k \rho_{1}\right)\right] K_{2}\left(q_{e} \rho_{1}\right) / \\
& K_{1}\left(q_{e} \rho_{1}\right)=2 q_{e} \rho_{0} I_{2}\left(q_{i} \rho_{0}\right) / \pi q_{i} \rho_{1} I_{1}\left(q_{i} \rho_{0}\right) .
\end{aligned}
$$

Here $J_{n}$ and $Y_{n}$ are Bessel functions, $I_{n}$ and $K_{n}(n=0,1)$ are modified Bessel functions; $k$ and $q_{i}$, are structural parameters of the system that have to be found; radius $\rho_{0}$ and $\rho_{1}$ are free (input) parameters; $q_{e}^{2}=-\beta_{e} / V_{0 x} \equiv d R_{s w}^{-2}(d P / d y) / V_{0}$. Normalized by radius $\left(\rho_{1}\right)$ structural parameters $k$ and $q_{i}$, (i.e. quantities $q_{i} \rho_{0}$ and $k \rho_{1}$ ) have to be determined from (4). The SW mode wave number, $k$, determines the number of FAC structures in the boundary region $\rho_{0}<\rho<\rho_{1}$; wave number $q_{i}$ characterizes the SW field structure in the inner region $\rho<\rho_{0}$. Dimensionless quantity $q_{e} \rho_{1}=\sqrt{ }\left(\beta \rho_{1}^{2} / V_{0}\right)$ is equal to $V_{0 E} / V_{0}$.

The FAC intensity, $j_{\|}$can be derived from the magnetic field potential $A$ (1) by the relationship $j_{\|}=-\mu_{0}^{-1}$ $\Delta A \equiv-\mu_{0}^{-1} B_{0} \Delta \varphi / V_{A}$. It can be found that

$j_{\|, g} \propto V_{0}(\Theta)\left(R_{b}\right)^{1 / 2} / \rho_{1} \lambda_{F A C}^{2}\left(\operatorname{IMF} B_{z}\right)$,

where $R_{b}$ is the plasma density in the boundary region, and scale $\lambda_{\text {FAC }}$ is the IMF $B_{z}$ dependent parameter. Note that FAC density $j_{\|, g}$ is inversely proportional to the squared scale $\lambda_{\text {FAC }}$. In the polar cap region, $\lambda_{\mathrm{FAC}, p c} \equiv 1 / q_{i}$, in Region 1 and Region 2 , FAC scale $\lambda_{\mathrm{FAC},} R 1+R 2 \equiv 1 / k$. Under negative IMF $B_{z}$, the scale of the polar cap radius $\rho_{0}$ increases and the size $\rho_{1}-\rho_{0}$ decreases, hence $\lambda_{\text {FAC, } p c}$ decreases, while the scale $\lambda_{\text {FAC, } R 1+R 2}\left(\sim\left(\rho_{1}-\rho_{0}\right)^{-1}\right)$ increases. Under positive IMF $B_{z}$ the polar cap radius, $\rho_{0}$ decreases and the size $\rho_{1}-\rho_{0}$ increases, hence $\lambda_{\mathrm{FAC}, p c}$ increases, while the scale $\lambda_{\text {FAC }, R 1+R 2}\left(\sim\left(\rho_{1}-\rho_{0}\right)^{-1}\right)$ decreases. The IMF $B_{x}$ and $B_{y}$ components introduce FACs that are proportional to

$j_{\|, B x(B y)}=. B_{x}\left(B_{y} \rho / \lambda_{\mathrm{FAC}}\left(\operatorname{IMF} B_{z}\right)\right)\left(R_{m}\right)^{1 / 2} / \rho_{1} \lambda_{\mathrm{FAC}}^{2}\left(\operatorname{IMF} B_{z}\right)$,

As follows from Eq. (6) the FAC induced by the IMF $B_{y}$ component is $\rho / \lambda_{\mathrm{FAC},} R 1+R 2$ times the FAC induced by the IMF $B_{x}$ component. Compared to Eq. (5) the IMF $B_{y}$ FAC magnitude is $V_{A, b} B_{y} / V_{0} B_{0}$ times the ground-state FAC intensity. Let us make some estimate of the ratio $V_{A, b} B_{y} / V_{0} B_{0}$. The Alfvén velocity at the magnetopause and in the boundary regions, of course, varies. On the basis of 3-year Cluster measurements, the phase velocity of MHD (kink-like) perturbations at the plasma sheet boundary layer (PSBL) is of the order of the local Alfvén velocity that is between 600 and $1400 \mathrm{~km} / \mathrm{s}$ (Grigorenko et al., 2007). In the nearEarth magnetotail the local Alfvén velocity is $\sim 400 \mathrm{~km} / \mathrm{s}$ 
(Runov et al., 2007 ${ }^{1}$ ). In the high-altitude polar cusp the Alfvén velocity is $200-800 \mathrm{~km} / \mathrm{s}$ (Grison et al., 2005). In the high-latitude boundary layer (HLBL) the local Alfvén velocity is about $350 \mathrm{~km} / \mathrm{s}$ at distance $10 R_{e}$ (Nykyri et al., 2006). The measured Alfvén speed in different boundary layers, like LLBL, HLBL, and the high-altitude cusp thus proves to be mostly in the interval $200-800 \mathrm{~km} / \mathrm{s}$. The plasma density in the polar cap region varies between $0.2-1 \mathrm{part} / \mathrm{cm}^{3}$, in the boundary region (LLBL), is by an order higher (1$10 \mathrm{part} / \mathrm{cm}^{3}$ ) (Nykyri et al., 2006). In the inner/polar cap region the Alfvén velocity is taken to be proportional to $\left(R_{m} / R_{p c}\right)^{1 / 2} V_{A, b}$, where $R_{b}$ and $R_{p c}$ are the plasma densities in the boundary region and in the polar cap, respectively. In our large-scale FAC simulation the Alfvén velocity, $V_{A . b}$, in the boundary region, is taken to be equal to $400 \mathrm{~km} / \mathrm{s}$. For convenience, the $R_{m} / R_{p c}$ ratio is assumed to be equal to 20 . The other quantities, IMF $B_{y}$ component, solar wind speed, $V_{0}$ and magnetopause magnetic field, $B_{0}$ are, in principle, known. Taking $V_{A}$ and $V_{0}$ to be equal to $400 \mathrm{~km} / \mathrm{s}$ and the magnetic field $B_{0}$ at the magnetopause to be of the order of $100 \mathrm{nT}$, the ratio $V_{A, b} B_{y} / V_{0} B_{0}$ thus rarely exceeds a value of 0.1 .

FAC simulations yield that the IMF $B_{x}$ component contribution to the large-scale FAC intensity is practically by an order less than the IMF $B_{y}$ component contribution. The IMF $B_{y}$ component contribution (proportional to the ratio $\rho / \lambda_{\mathrm{FAC}, R 1+R 2}>1$ ) may, however, constitute tens or even more percents of the large-scale FAC ground-state magnitude.

The SW mode FAC system structure depends on external parameters: $q_{e}, V_{0}$, IMF components, $B_{x}, B_{y}$ and $B_{z}$, and internal parameters: $B_{0}$, plasma density distribution $R_{m}$, and radius $\rho_{0,1}$. Radius $\rho_{0}$ and radius $\rho_{1}$ are free parameters of the proposed SW mode FAC model. Actually, they have to be related to the polar cap boundary and the lowest latitude of the disturbed magnetic field lines. Radius, $\rho_{0(1)}$ is thus closely connected with the sign and magnitude of the IMF $B_{z}$ component. Following experimental evidences, under positive IMF $B_{z}$ conditions, the polar cap boundary (that corresponds to radius $\rho_{0}$ ) is close to the $80^{\circ}$ magnetic latitude; under negative IMF $B_{z}$ the polar cap boundary is $\sim$ at $75^{\circ}$ and lower. The external radius $\rho_{1}$ also depends on the solar wind and IMF parameters. Radius $\rho_{1}$ and plasma density variations in the boundary region $\rho_{0}<\rho<\rho_{1}$ have to also be treated as a function of the previous state of the magnetosphere and thus these parameters reflect the dynamic history of the Earth's magnetosphere. Their actual magnitudes seem to be connected with the inner magnetosphere conditions, i.e. they are inertial ones. In our analysis we assume that radius $\rho_{1}$ and plasma density distribution $R_{m}$ are constants. For convenience, radius $\rho_{1}$ is assumed to correspond to $60^{\circ}$ (magnetic latitude).

\section{Large-scale FAC system - a ground state}

A ground state of the large-scale FAC system, consisting of six cells, can be recognized if one considers all the IMF components to be nearly equal to zero. In our FAC calculations the magnitude of the Earth's magnetic field at the magnetopause $B_{0}$ is taken to be equal to $120 \mathrm{nT}$, the solar windmagnetosphere interaction occurs at radial distance $r=10 R_{e}$, the solar wind velocity $V_{0}$ varies, or is equal to $400 \mathrm{~km} / \mathrm{s}$, the tilt angle $\Theta$ is assumed for summer, equinox and winter conditions. Actually, the Earth's magnetic field at the magnetopause $B_{0}$ is dependent on solar wind pressure variations, thus the solar wind velocity and the $B_{0}$ magnitude are in fact interrelated. When the solar wind velocity increases, $B_{0}$ also increases. For comparison with experimental data obtained mainly from low-altitude satellites, a map of the FAC intensity along the magnetic field tube to ionospheric heights is required. Due to the flux conversion $(\boldsymbol{B} . \boldsymbol{S}=$ const $)$ the FAC intensity converges, as well. The FAC intensity depends on radial distance $r$ (from the Earth's surface) as $\left(r_{\mathrm{FAC}} / r_{I}\right)^{3}$, where $r_{\mathrm{FAC}}$ is the radial distance of the FAC source and $r_{I}$ is the ionosphere shell distance $\left(r_{I} \cong R_{e}\right)$. Thus, at ionosphere heights $\left(r_{I} \cong R_{e}\right)$ the FAC intensity will increase roughly by three orders compared to its density at the FAC source distance $\left(r_{\mathrm{FAC}} \cong 10 R_{e}\right)$. In Fig. 2 the FAC intensity is evaluated at ionospheric heights, taking into account the magnetic flux conservation principle. The polar cap boundary is taken to be equal at $78^{\circ}$ magnetic degrees and corresponds to IMF $B_{z}$ equal to $2 \mathrm{nT}$. The IMF $B_{x}$ and $B_{y}$ components are set to zero.

The six-cell FAC ground state given in Fig. 2 depicts the main experimental features of the large-scale FAC structure satellite observations. FAC intensity distribution possesses maxima and minima close to the central dawn-dusk line, and the absolute values of the Region 1 FAC intensity exceed the absolute values of the Region 2 FAC intensity. The FAC intensity distribution possesses a maximum that is below but close to $1 \mu \mathrm{A} / \mathrm{m}^{2}$. Note that the SW mode FAC model yields a quantitative relationship between the FAC intensity and the solar wind velocity $V_{0}$ and the boundary plasma density $R_{b}$. According to the model (Eq. 5) the FAC density is proportional to solar wind velocity $V_{0}$ and boundary plasma density $\left(R_{b}\right)^{1 / 2}$.

The IMF influence on the large-scale FAC system is examined further. The six-cell FAC ground state still exists under various IMF conditions. Considering the IMF $B_{y}$ component to be equal to zero (while IMF $B_{x}$ and $B_{z}$ components are variable), the zero-frequency SW mode FAC model again yields an existence of a six-cell FAC structure, as was previously discovered (Nenovski, 2003). The relevant IMF components are easily transformed from the GSE to GSM coordinate system by a simple rotation about the $\mathrm{X}$-axis. This means that the IMF components $B_{x}\left(B_{y}\right)$ are influenced by the IMF $B_{z}$ component under summer/winter (equinox) conditions. Because the IMF $B_{y}=0$, an effective $B_{y}$ component emerges 
that depends on the IMF $B_{z}$ component only. Three values of the IMF $B_{z}$ component are chosen: $B_{z}=2 \mathrm{nT}, B_{z}=10 \mathrm{nT}$, and $B_{z}=-10 \mathrm{nT}$. For IMF $B_{z}=2 \mathrm{nT}$ the polar cap boundary is taken to be equal to 78 degrees, for $B_{z}=10 \mathrm{nT}$, this boundary is 82 degrees, and for $B_{z}=-10 \mathrm{nT}$, the polar cap boundary is 74 degrees. Figure $3 a, b$ depicts a six-cell FAC system where the IMF $B_{y}$ is set to zero under equinox conditions.

The FAC structure demonstrates variations of the FAC density in the polar cap depending on the IMF $B_{z}$ component magnitudes. When the IMF $B_{z}$ becomes negative, the FAC intensity in the polar cap decreases and under strong negative IMF $B_{z}$ conditions (IMF $B_{z}$ equals to $-10 \mathrm{nT}$ ) the initial six-cell FAC structure practically evolves into a four-cell FAC structure that occupies the boundary region $\left(\rho_{0} \leq \rho \leq \rho_{1}\right)$ only. Inversely, when the IMF $B_{z}$ is positive the 6-cell FAC structure emerges clearly and the polar cap FAC (dipole) structure is of high intensity. Region 1 and Region 2 FAC systems thus co-exist.

In Fig. 3a, b the boundary plasma density is chosen to be invariable. Note that the induced FAC intensity variations are a function not only of the boundary region size, but also of the plasma density variations in the boundary regions under a different sign of the IMF $B_{z}$.

\section{$I M F B_{y}$ effects on the large-scale FAC system}

Further, in our analysis we will be concerned with the effects of IMF $B_{y}$, as well as the possible changes caused by the negative/positive IMF $B_{z}$ component on the FAC system.

Examples of large-scale FAC structures as a solution for the Eq. (2) are given in Fig. 4a-c. Figures $4 a-c$ illustrate large-scale FAC structure as influenced under negative, zero and positive IMF $B_{y}$. The IMF $B_{z}$ and $B_{x}$ are taken to be positive and equal to $2 \mathrm{nT}$. The inner radius $\rho_{0}$ is chosen to be at 78 degrees (that corresponds to northward IMF conditions), the outer radius $\rho_{1}$ corresponds to 60 degrees. The other parameters are: the solar wind velocity is $400 \mathrm{~km} / \mathrm{s}$, the boundary density is 10 particles $/ \mathrm{cm}^{3}$, the number density in the tail lobe is 20 times less. Figures $4 a-c$ reveal the effects as expected from the DPY current system: during positive IMF $B_{y}$ the Region 1 FAC system from the dawn sector is extended across the noon sector. This FAC attaches the dusk part of the FAC system in the polar cap. On the other hand, the Region 1 FAC system from the dusk sector (of inverse polarity) continues into the Region 2 FAC system from the dawn sector. This FAC structure modification clearly emerges only under stronger IMF $B_{y}$ magnitudes (when the IMF $B_{y}$ module exceeds $2 \mathrm{nT}$ ). It seems that the six-cell FAC structure would evolve into two FAC "spirals" of inverse polarity where both spirals start from the polar cap regions. If the IMF $B_{y}$ sign reverses, the sense of the FAC "spirals" also reverses. In the midnight sector the dawn (dusk) Region 1 FAC structure goes continuously to the dusk (dawn) Region 2 FAC cell.

Under equinox conditions the FAC intensities from both hemispheres are expected to be equal to each other. The
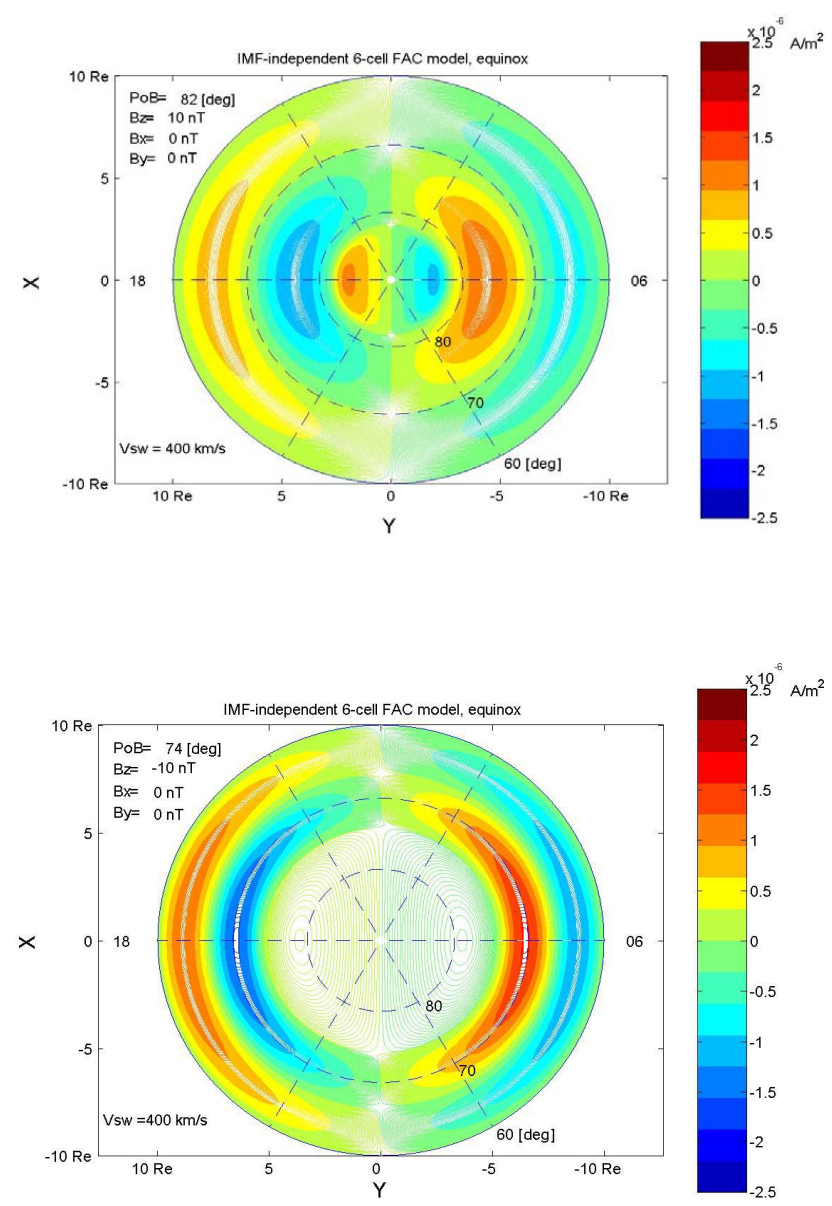

Fig. 3. FAC density variations in the polar cap (the dipole FAC structure), depending on the IMF $B_{z}$ component magnitudes, are illustrated. This figure depicts a six-cell, large-scale FAC system under equinox conditions where the IMF perpendicular component, $B_{y}$, is assumed to be equal to zero. The coordinates are the same as in Fig. 2. Under negative IMF $B_{z}$ conditions (IMF $B_{z}$ equals to $-10 \mathrm{nT}$ ) the initial six-cell FAC structure practically evolves into a four-cell FAC structure that occupies latitudes $60^{\circ}-74^{\circ}$ magnetic degrees only. Inversely, when the IMF $B_{z}$ is positive (IMF $B_{z}$ equals to $10 \mathrm{nT}$ ) the six-cell FAC structure clearly emerges where the polar cap (dipole) FAC structure is of high intensity.

equipartition principle implies that the FAC intensities obtained so far should be divided by two because of the two possible propagation directions of these FAC - away and to the Earth. The cylindrical geometry of our FAC model is symmetrical and hence it cannot incorporate the summer/winter effects. Nevertheless, qualitative considerations and rough estimates of the FAC intensity under different season conditions are still possible. Indeed, the actual geometry of the solar wind flow around the high-latitude Earth's magnetic field lines is quite different under summer and winter conditions. In summer the solar wind flow encounters the Earth's magnetic field lines at angles close to the right angle (a tail lobe interaction is expected). In winter, the same 

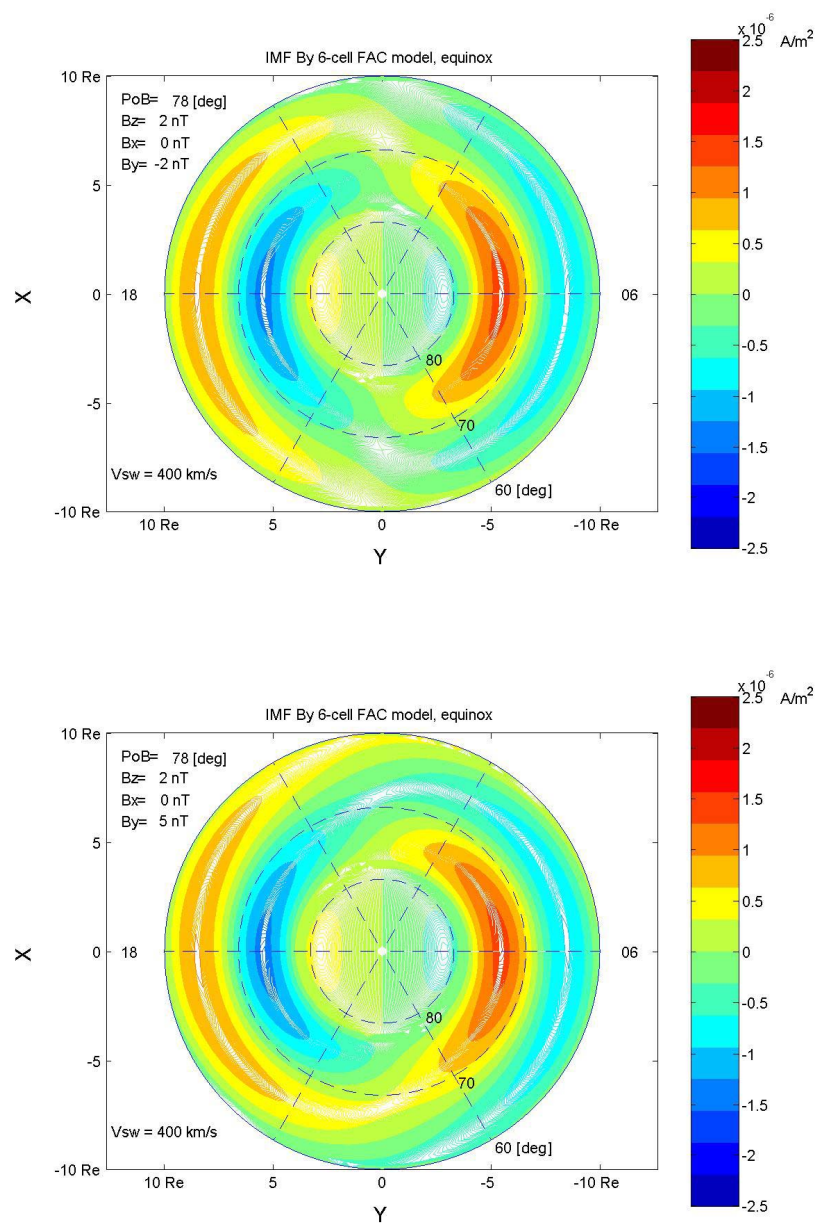

Fig. 4a. Illustrate how a negative/positive IMF $B_{y}$ component influences the SW mode FAC structure. During positive IMF $B_{y}$, the Region 1 system from the dawn sector is extended across the noon sector. On the other hand, the Region 1 system from the dusk sector (of inverse polarity) continues into the Region 2 system from the dawn sector. When the IMF $B_{y}$ module well exceeds $2 \mathrm{nT}$, the sixcell FAC structure evolve into two FAC "spirals" of inverse polarity. When the IMF $B_{y}$ component reverses its sign, the sense of the FAC "spirals" also reverses. In the midnight sector the dawn (dusk) Region 1 structure goes continuously to the dusk (dawn) Region 2 cell. The IMF $B_{z}$ component is set to $-10,2$ and $10 \mathrm{nT}$. Figure $4 \mathrm{~b}$ is for IMF $B_{z}=-10 \mathrm{nT}$, and $4 \mathrm{c}-$ for IMF $B_{z}=10 \mathrm{nT}$.

high-latitude magnetic field lines create with the solar wind direction an angle well below angle $\pi / 2$; the solar wind velocity thus may not encounter effectively the high-latitude near the Earth's magnetic flux tube. A decrease in the FAC intensity, additional to factor $\cos (\Theta)$ thus will emerge. Note only the nonzero projection of the solar wind velocity vector on the plane perpendicular to the Earth's magnetic fluxes is responsible for the large-scale FAC structure generation. Qualitatively, the FAC intensity difference between summer and winter conditions may be assumed to be proportional to the ratio of the high-latitude Earth's magnetic flux volume
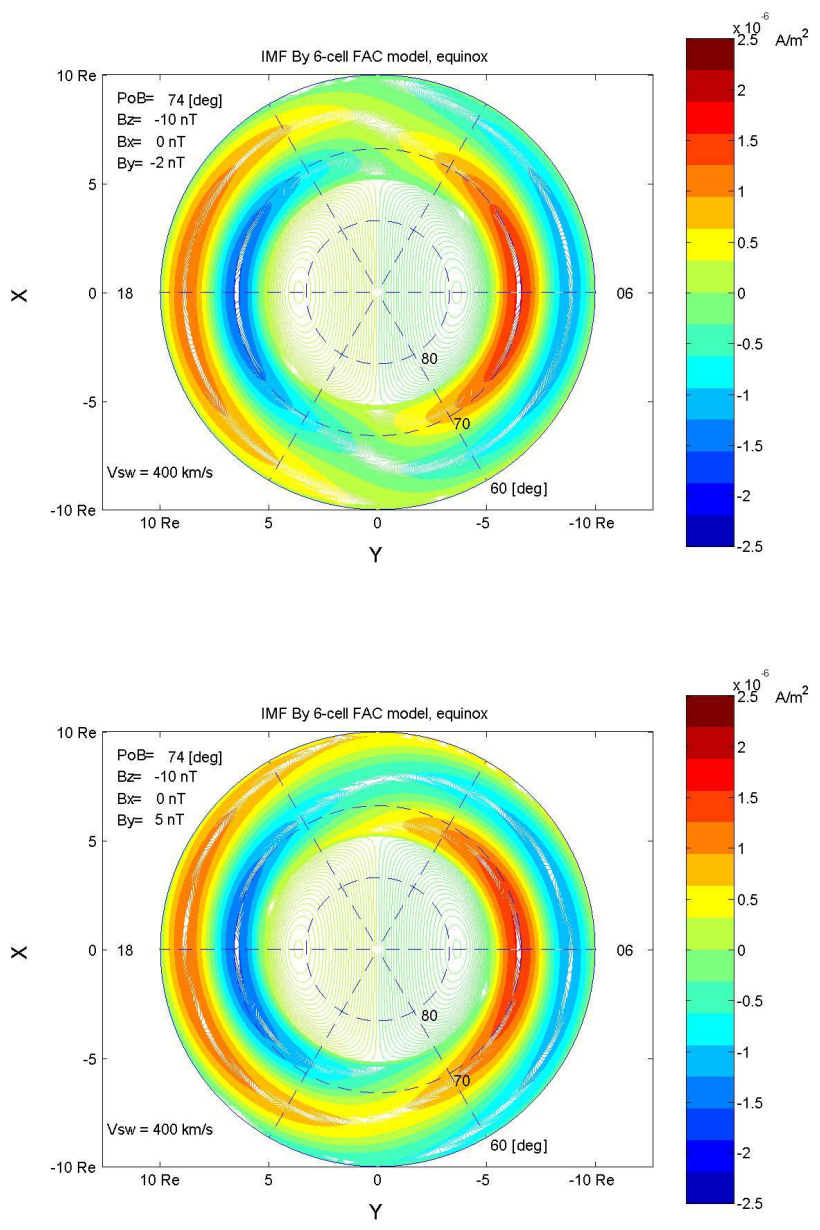

Fig. 4b. Continued.

encountered by the solar wind flow. The two effects result in a FAC intensity difference between summer and winter. In comparison to the equinox conditions the FAC intensity for summer becomes 1.6 times greater. Under winter conditions the FAC intensity is, however, approximately three times less than in equinox. Figure 5a, b characterizes the six-cell FAC structure under summer and winter conditions.

The SW mode FAC model also reveals some difference that depends on the magnitude of the IMF $B_{z}$ component. This difference comes from the different sign of the IMF $B_{z}$ projection on the equatorial plane of the Earth's magnetic field dipole axis. Hence, the contribution of the IMF $B_{y}$ effect, i.e. the IMF $B_{y}$ FAC intensity may be different.

\section{Comparison with experimental data}

The modelled FAC intensity $j_{\|}$at ionosphere heights becomes of the order of $\sim(1 \div 2) .10^{-6} \mathrm{~A} / \mathrm{m}^{2}$ (under equinox and summer conditions). Such values are comparable with those obtained from TRIAD satellite magnetometer data. For the period of July 73-October 74 and geomagnetic 

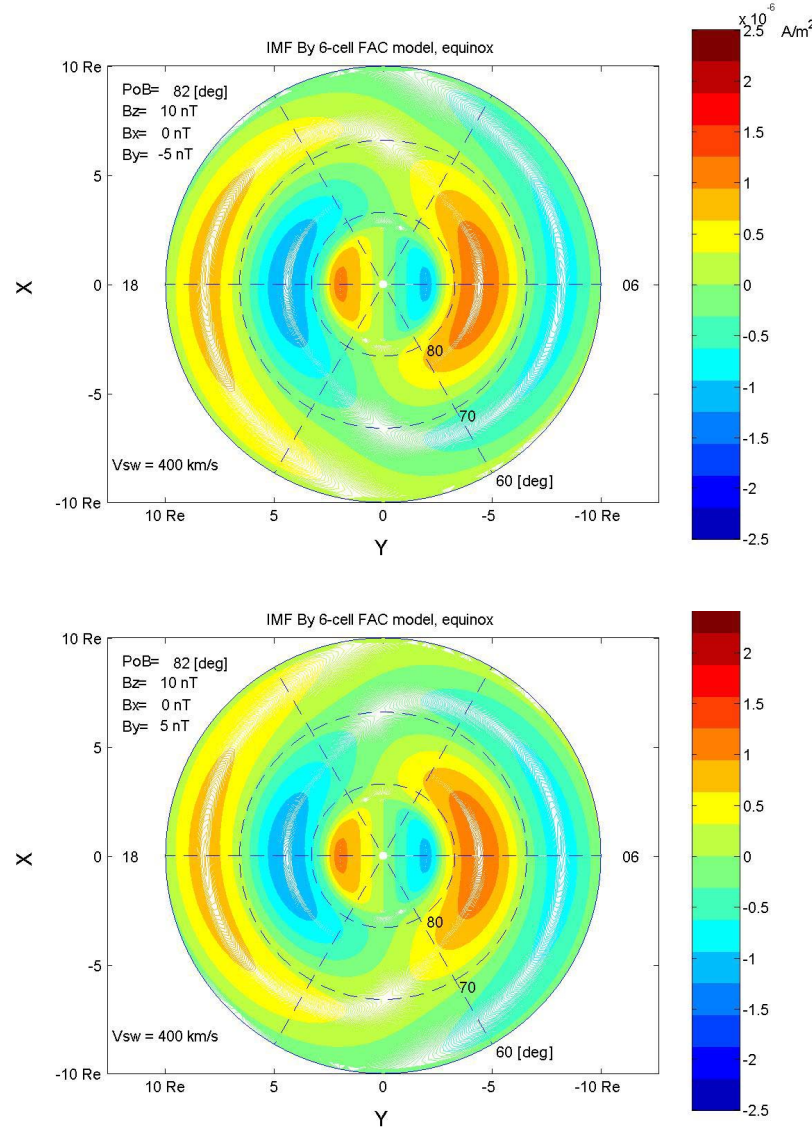

Fig. 4c. Continued.

conditions $2^{-} \leq K p \leq 4^{+}$the Region 1 FAC maximum intensity amplitude was $2.0(1.3) \mu \mathrm{A} / \mathrm{m}^{2}$ and the Region 2 FAC maximum intensity amplitude was $\left.1.0(0.6) \mu \mathrm{A} / \mathrm{m}^{2}\right)$ at the morning (evening) sectors (Iijima and Potemra, 1978). The first statistical picture of FACs contains intense FAC structures (of $\sim 1.0 \mu \mathrm{A} / \mathrm{m}^{2}$ ) in the nightside sector, not predicted in our zero-frequency MHD surface mode model. This is because Iijima and Potemra's FAC picture integrates FAC structure intensifications during dynamic processes, like magnetospheric (sub)storms released under negative IMF $B_{z}$ conditions, where merging/reconnection-like processes occupy the evening-midnight sector of the polar ionosphere.

Recently, precise magnetic field measurements on board two satellites, Magsat and Oersted, have demonstrated new properties in the physics of steady-state FAC systems. Using data from Magsat and Oersted high-precision magnetic measurements the satellite orbits have been sorted on the basis of strict criteria to ensure steady IMF and solar wind conditions at the time of the polar pass (Stauning, 2003). Experimental evidences and results have been summarized by Stauning (2003). Thus, the proposed large-scale FAC structure model could be tested on the observed FAC systems under various IMF conditions - positive and negative IMF $B_{z}$,
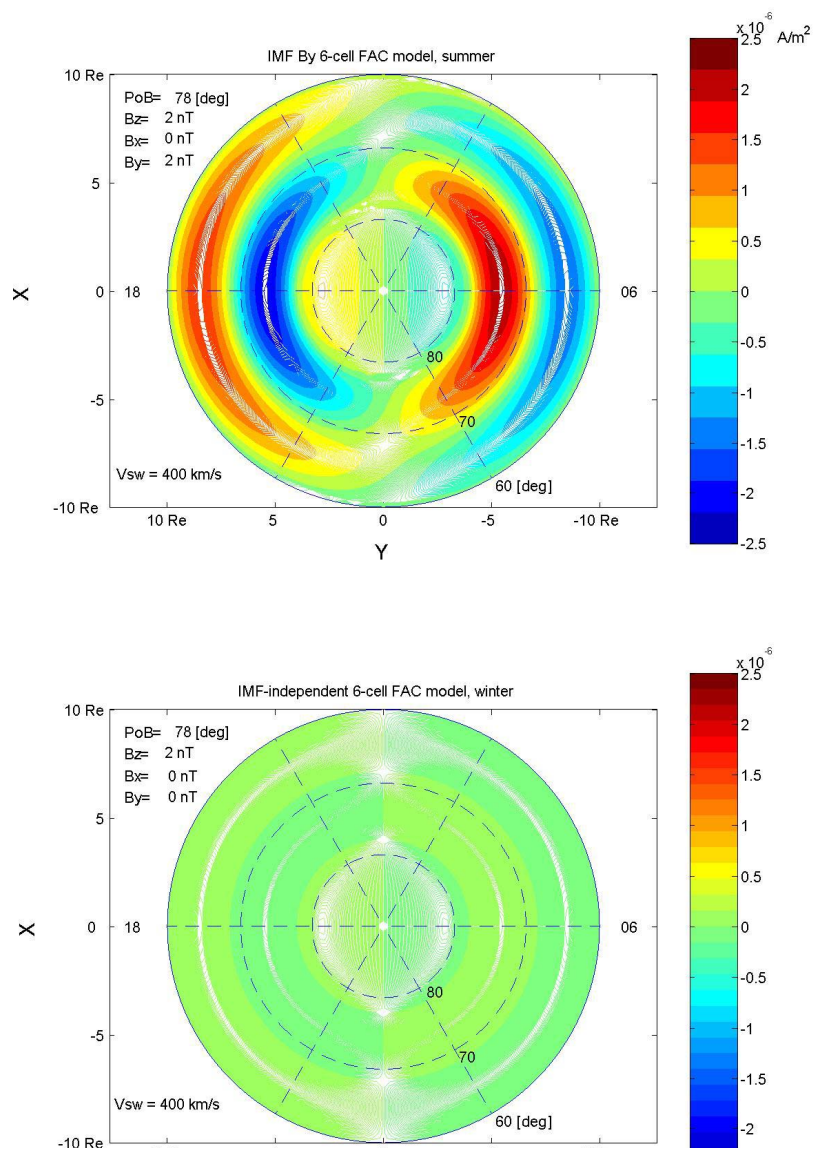

Fig. 5. Figure 5a, b characterize the six-cell FAC structure under summer and winter conditions. In comparison to equinox conditions (not shown) the FAC intensity in summer becomes 1.6 times greater. Under winter conditions the FAC intensity is, however, approximately three times less than in equinox.

dominant IMF $B_{y}$ component, summer/winter, or equinox, etc. The NBZ FAC intensity over the northern polar cap attains a magnitude of $1 \mu \mathrm{A} / \mathrm{m}^{2}$ and more when there is the summer season and the IMF $B_{z}$ component is within inequalities: $10 \mathrm{nT}<\mathrm{IMF} B_{z}<20 \mathrm{nT}$ (Stauning, 2002). Our results on FAC density magnitudes during northward IMF and summer conditions (Figs. 3 and 5) are consistent with such experimental evidences. The FAC model, however, does not properly reveal the noon- asymmetry of the NBZ FAC current system in the polar cap region. The observed NBZ FAC system is, on average, slightly shifted toward the sunward/noon side of the polar cap. The simulated polar cap FAC system is found to be centred symmetrically at the central dawn-dusk line, i.e. it is axial-symmetric. Such an inconsistency might be explained by the fact that the Earth's magnetic field lines on the dayside (the cusp region) are more compressed (from the solar wind pressure) than those on the nightside. In summer more magnetic field lines are exposed to a direct impact from the solar wind flow, hence the compression effect on 
them has to be enhanced. Thus, both magnetic flux and respective FAC density on the noon part of the polar cap should be more enlarged compared to that on the nightside.

Differences in the FAC intensity due to the season and the IMF $B_{z}$ sign are examined by Stauning (2003). When the IMF $B_{y}$ component is close to zero $\left(-2 \mathrm{nT}<\mathrm{IMF} B_{y}<2 \mathrm{nT}\right)$ and the IMF $B_{z}$ component is variable, the FAC density becomes its maximum either in the polar cap (the IMF $B_{z}$ is then positive ( $>5 \mathrm{nT}$ ), or in the Region 1 FAC system (the IMF $B_{z}$ is then negative, $-10 \mathrm{nT}<\operatorname{IMF} B_{z}<-5 \mathrm{nT}$ ). In the first case the polar boundary of the Region 1 FAC system is at $80^{\circ}$ magnetic latitude, in the second case (of negative IMF $B_{z}$ ) the Region 1/2 FAC systems occupy a belt of some 15 degrees, i.e. between $60^{\circ}-75^{\circ}$ magnetic latitude. By an exact choice of the inner radius $\rho_{0}$ (the polar cap boundary), the proposed FAC system model (Figs. 2-5) reconciles the main features of the observed FAC distribution (under equinox and summer/winter conditions) and their maxima around the central dawn-dusk line.

The IMF $B_{y}$ influence on the FAC system has been also examined. Stauning (2003) has displayed the FAC structure under equinox and negative IMF $B_{z}\left(-5 \mathrm{nT}<\mathrm{IMF} B_{z}<-2 \mathrm{nT}\right)$ conditions. Two solar wind velocity regimes are considered: $200<V_{0}<400 \mathrm{~km} / \mathrm{s}$ and $400<V_{0}<900 \mathrm{~km} / \mathrm{s}$. The FAC densities have their extrema in the Region 1 FAC system and again at the dawn-dusk central line, and are proportional to the solar wind velocity magnitude. The FAC structure is practically a four-cell one. A single downward/upward polar cap FAC cell centred at noon and at $80^{\circ}$, or at $80^{\circ}-85^{\circ}$ magnetic latitudes under positive/negative IMF $B_{y}$, may additionally be recognised. Another polar cap FAC cell of inverse polarity, however, cannot be identified clearly. Having in mind our findings of the emergence of spiral-like FAC structures under dominant IMF $B_{y}$, such an asymmetry in the polar cap FAC structure seems not to have experimental verification.

When the interplanetary magnetic field (IMF) was either near zero, or different from zero (with IMF $B_{z}>0$ and IMF $B_{z}<0$ ), a persistent pair of FACs of inverse polarity flowing poleward of the Region 1 FAC system and placed in the central area of both the southern and northern polar caps has been discovered (Papitashvili et al., 2001, 2002; Weimer, 2001b; Stauning, 2002). The distribution of these currents under IMF orientation resembles the dayside NBZ system, with the downward/upward currents at post-noon/pre-noon hours. Hence, the polar cap FAC system polarity is opposite to that of the Region 1 FAC system.

Let us quantitatively compare the SW mode FAC system to empirical large-scale FAC models based on Oersted and Magsat satellite data. A steady-state FAC distribution for a different IMF clock angle has been derived (Papitashvili et al., 2001, 2002). Analyzing the IMF $B_{y}=0$ case, the FAC intensity usually is maximum in the Region 1 FAC system, except when the IMF $B_{z}>0$ and in the southern (summer) hemisphere case. The FAC intensity maximum in the Region 1 FAC system in the Southern (Northern) Hemisphere varies from $+0.56(0.35)$ to $-0.51(0.29) \mu \mathrm{A} / \mathrm{m}^{2}$ under southward IMF $B_{z}$ conditions in the morning (sign + ) and evening (-) sectors. Signs +/- refer to the downward/upward FACs in the morning/evening sectors. Under northward IMF $B_{z}$ conditions the FAC intensity in Region 1 are weaker: from 0.07 to $-0.15 \mu \mathrm{A} / \mathrm{m}^{2}$ in the Northern (winter) Hemisphere. Surprisingly, the upward FAC intensity in the Southern (summer) Hemisphere has its absolute maximum in the polar cap: $-0.3 \mu \mathrm{A} / \mathrm{m}^{2}$. The downward FAC intensity, however, persists in the Region 1 FAC system: $+0.15 \mu \mathrm{A} / \mathrm{m}^{2}$. Satellite observations have revealed a strong control of the IMF $B_{y}$ component on the polar cap FAC intensity in the summer hemisphere. The average magnitude of Region 1 FAC varies between 0.1 and $0.56 \mu \mathrm{A} / \mathrm{m}^{2}$.

An evaluation of the total FAC flowing in and out of the ionosphere can be performed choosing all FAC cells of one polarity and integrating the FAC intensity. As a rough estimate, the total FAC of one polarity (depending on the season conditions) amounts to $10^{5}-10^{6} \mathrm{~A}$, depending on solar wind, IMF and seasons conditions. The result is in keeping with experimental evidences.

The negative/positive IMF $B_{z}$ is suggestive for different plasma dynamics and densities in the boundary regions; this results in a different amount of available FAC carriers within them. Accordingly, the SW mode FAC model yields a FAC intensity distribution depending on the boundary/polar cap density ratio. The SW mode FAC model is also sensitive to the solar wind parameter variations. The Papitashvili et al.'s (2002) result based on Oersted observations represents an average FAC picture depending on the IMF clock angle and is irrespective of the solar wind velocity variations. Nevertheless, the six-cell FAC system (one pair FAC structure in the polar cap and two pairs of FACs in the boundary (auroral) regions) predicted by the SW mode FAC model, may find their counterparts in the Oersted satellite observations. The FAC intensities calculated from both the SW mode FAC model and Oersted data are of comparable magnitudes.

The SW mode FAC model thus yields qualitatively and quantitatively a good correspondence with experimental results for reasonable values of the solar wind velocity and IMF parameters. The correspondence consists of: (i) two pairs of FAC structures of inverse polarity that emerge within the boundary region $\left(\rho_{0}<\rho<\rho_{1}\right)$. They may be interpreted as Region 1 and Region 2 FAC structures observed in the auroral region; (ii) a pair of FAC structures of inverse polarity in the inner region $\left(\rho<\rho_{0}\right)$. This polar cap FAC structure exists under the arbitrary sign of the IMF $B_{z}$ component but its intensity is much higher under northward IMF $B_{z}$. The latter may be considered as tail-lobe FACs; and iii) reasonable magnitudes of the FAC intensity distribution. Therefore, the SW mode FAC model explains the structural characteristics of the observed large-scale FAC systems and quantifies the FAC intensities depending on the solar wind and IMF parameters. A detailed comparison between experimental results and FAC system models, however, is quite 
desirable, especially for the cases with a positive IMF $B_{z}$ component. Further, the effects of the plasma density change in the boundary region $\left(\rho_{0}<\rho<\rho_{1}\right)$ during positive/negative IMF $B_{z}$ on large-scale FAC distribution need to be precisely estimated.

In conclusion, a dipole-like (two cells of inverse polarity) FAC system in the inner region $\rho<\rho_{0}$ (polar cap, or tail lobe) and four (two pairs) FAC cells of inverse polarity (that encircle the dipole FACs), localized in the boundary region $\rho_{0}<\rho<\rho_{1}$, can co-exist (Nenovski, 2003). It is worth noting that in the boundary region solutions of Eq. (4) with a lower number of FAC cells (less than 4) cannot be found, i.e. at least 4-cell FACs (Region 1 and Region 2 FACs) in the boundary region co-exist with a pair of (dipole) FACs in the polar cap. These crescent-like FAC regions (belts) encircle azimuthally the dipole/central FAC structure. The complete FAC system is flowing (along the magnetic field lines) toward the Earth. The FAC intensities in all regions have their maxima/minima around the line perpendicular to the solar wind flow $\left(\boldsymbol{V}_{0}\right)$, going through the locus of the circle of interaction.

The results derived from the SW mode FAC model suggest that a common nonlinear MHD mechanism of the solar wind-magnetosphere interaction can only support a six-cell large-scale FAC structure. This six-cell structure exists even under zero IMF conditions. An inclusion of IMF components reveals the principal features inherent either to the NBZ FAC system that exists under positive IMF $B_{z}$, or to the Region 1 and Region 2 FAC system under stable negative IMF $B_{z}$ conditions. An inclusion of the IMF $B_{y}$ component produces a Region 1 FAC extension over the noon sector that corresponds to the DPY system. Obviously, the proposed largescale SW mode FAC structure is consistent with processes (viscous and/or merging ones) that have been suggested in previous FAC models.

The above general conclusions need some further remarks. Even in steady-state conditions the primary six-cell FAC system may be easily destroyed toward the Earth's ionosphere. First, due to the Earth's magnetic field line conversion, the FAC intensity grows and under appropriate conditions can easily exceed a magnitude of $10^{-6} \mathrm{~A} / \mathrm{m}^{2}$. Such FAC intensity magnitudes may be sufficient for plasma instability outbursts. An instability of large-scale FAC may result in FAC structure changes also, e.g. the appearance of multiple FAC structures of a smaller size. As a result, the intensity of the FAC structures of medium size will exceed those of the large-scale ones. Second, the FAC Joule dissipation rate in the ionosphere will be qualitatively and quantitatively different with and without multiple FAC structures. Satellite observations of FAC structures and the associated convection electric field in the polar ionosphere have already confirmed the existence of more intense multiple FAC structures and stronger convection electric fields (e.g. Ohtani et al, 1994; Eriksson et al., 2002). Existing convection electric field models (e.g. Weimer, 2001a, b; Papitashvili and Rich,
2002) have lacked the ability to simulate more intense structures of medium scales, i.e. those of several hundreds of $\mathrm{km}$, observed, say, by the Astrid-2 satellite.

An emergence of structures of medium scales $a$ in the large-scale FACs regions and in the global convection electric field in the polar/polar cap ionosphere may be a direct consequence of the FAC pattern instability treated by Nenovski et al. (2003). Without quoting the formalism of the structural instability that follows from the FAC intensity increase toward the ionosphere, the FAC pattern formation begins at

$j_{\|, \text {crit }} a \geq 0.2\left(v_{T} / v_{T o}\right)[\mathrm{A} / \mathrm{m}]$

where $v_{T o}$ is the "thermal" velocity of the FAC carriers that corresponds to plasma temperature $T_{0}$ of $0.1 \mathrm{eV}$. The FAC threshold value intensity magnitude increases with the "thermal" velocity $v_{T}$ of the FAC carriers. The obtained threshold value (7) can be expressed by the associated convection electric field $\boldsymbol{E}$ that is mapped along the magnetic field lines to the ionosphere heights. Depending on the ionospheric Pedersen and Hall conductances, the threshold values, $E_{\text {thresh }}$ will be of different magnitude. In its simplest form (homogeneous Pedersen and Hall conductances) the following relationship between $E_{\text {thresh }}$ and $j_{\| \text {,crit }}(m, n)$ can be derived from Eq. (1):

$E_{\text {thresh }}=j_{\|, \text {crit }}(m, n) / \Sigma_{p}$.

The ionospheric conductances are greatest in the auroral regions compared with those in the polar cap. Hence, multiple FAC structures can be easily excited within the auroral oval. Taking the Pedesen conductance $\Sigma_{p}$ values of $10 \mathrm{~S}$ (a modest value) we found that a convective electric field magnitude of $20 \mathrm{mV} / \mathrm{m}$ can initiate multiple FAC/electric field structures. Such values are often detected on board the Astrid satellite (Eriksson et al., 2002). Note that the existence of the electric fields of magnitude $20 \mathrm{mV} / \mathrm{m}$, however, does not necessarily involve the formation of multiple FAC structures. In terms of the Pedersen current, the necessary condition is that $E_{\text {thresh }} \Sigma_{p}$ should exceed the critical value of $0.2 \mathrm{~A} / \mathrm{m}$ (see Eq. 7).

\section{Consequences and conclusion}

The principal issues that follows from the suggested SW mode FAC model are i) the large-scale FAC intensity and distribution in the polar ionosphere can be quantified provided that the solar wind, IMF and magnetospheric (polar cap/ and inner plasma sheet boundaries) parameters are known. Using these parameters as input parameters large-scale FAC characteristics in the polar regions can be derived and compared to available observational data.

The existence is predicted for ground-based six-cell FAC based on a nonlinear surface wave excitation mechanism at the solar wind-magnetosphere boundaries. The six-cell FAC 
structure exists irrespective of the sign of the IMF $B_{z}$ component. The main peculiarity of this mechanism is the coexistence of two pairs of FAC structures of opposite polarity (that correspond to Region 1 and Region 2 FAC), co-existing with a pair (dipole) of FAC structures over the polar cap. The SW concept of large-scale FACs also provides reasonable magnitudes of the morning and dusk part of the largescale Region 1 and 2 FACs, depending quantitatively on the solar wind and IMF parameters, the Earth's dipole axis orientation, etc.

Comparison with high-precision magnetic observations from Magsat and Oersted satellites gives a good correspondence between the observed FAC structures under steadystate conditions (Stauning, 2002, 2003) and those modelled by the zero-frequency SW mode excitation mechanism.

Main factors that can distort the predicted six-cell FAC structure are i) the indispensable intensification of the largescale FAC density and ii) the transition from high to low $\beta$ plasma conditions along their propagation direction. Both factors could facilitate FAC structures of different scales at appropriate FAC intensity threshold values. FAC pattern formation processes expected at ionosphere heights could be the most favourable mechanism of periodic FAC structures observed in high-latitude regions.

Large-scale FAC structure and associated FAC pattern formation processes being controlled by the solar wind and IMF conditions generate an associated electric potential distribution in the polar ionosphere regions and determine the Joule dissipation rate.

Acknowledgements. The author would like to express his thanks to J. Watermann for providing publications and proceeding materials from DMI, Copenhagen on magnetometric measurements on Magsat and Oersted satellites. The author also thank to both referees for their valuable criticism and comments that help to improve all issues from this study. This study was partly supported by the NSF Bulgarian Grant ES-1502/2005.

Topical Editor W. Kofman thanks J. Watermann and V. Papitashvili for their help in evaluating this paper. He also thanks F. Lefeuvre for his help in evaluating this paper.

\section{References}

Akasofu, S.-I., Ahn, B.-H., and Kisabeth, J.: Distribution of fieldaligned currents and expected magnetic field perturbations resulting from auroral currents along circular orbits of satellites, J. Geophys. Res., 85, 6883-6887, 1980.

Boyle, C. B., Reiff, P. H., and Hairson, M. R.: Empirical polar cap potentials, J. Geophys. Res., 102, 111-125, 1997.

Christiansen, F., Papitashvili, V. O., and Neubert, T.: Seasonal variations of high-latitude field-aligned currents systems inferred from Oested and Magsat observations, J. Geophsy. Res., 107(A2), 1029, doi:10.1029/2001JA900104, 2002.

Eriksson, S., Blomberg, L. G., and Weimer, D. R.: Comparing a spherical harmonic model of the global electric field distribution with Astrid-2 observations, J. Geophys. Res., 107(A11), 1391, doi:10.1029/2002JA009313, 2002.
Erlandson, R.E., Zanetti, L. J., Potemra, T. A., Bythrow, P. F., and Lundin, R.: IMF $B_{y}$ dependence of region 1 Birkeland currents near noon, J. Geophys. Res., 9, 9804-9814, 1988.

Friis-Christensen, E., Kamide, Y, Richmond, A. D., and Matsushita, S.: Interplanetary magnetic field control of high-latitude electric fields and currents determined from Greenland magnetosmeter data, J. Geophys. Res., 90, 1325-1338, 1985.

Grigorenko, E. E., Sauvaud, J.-A., and Zelenyi, L. M.: Spatialtemporal characteristics of the beamlets in the plasmasheet boundary layer of magnetotail, J. Geophys. Res., 112, AO5218, doi:10.1029/2006JA011986, 2007.

Grison, B., Sahraoui, F., Lavraud, B., Chust, T., CornilleauWehrlin, N., Reme, H., Balogh, A., and Andre, M.: wave particle interactions in the high-altitude polar cusp: a Cluster case study, Ann Geophys., 23, 3699-3713, 2005.

Iijima, T. and Potemra, T.: The amplitude distribution of fieldaloigned currents at northern high latitudes observed by Triad, J. Geophys. Res., 83, 1109-1114, 1976a.

Iijima, T. and Potemra, T.: Field-aligned currents in the dayside cusp observed by Triad, J. Geophys. Res., 81, p. 5971, 1976 b.

Iijima, T. and Potemra, T.A.: Large-scale characteristics of fieldaligned currents associated with substorms, J. Geophys. Res., 83, 599-615, 1978.

Iijima, T., Potemra, T. A., Zanetti, L. J., and Bythrow, P. F.: Largescale BIrkeland currents and the dayside polar region during strongly northward IMF: A new Birkeland current system, J. Geophys. Res., 89, p. 7441, 1984.

Kan, J. R. and Lee, L. C.: Energy coupling function and solar windmagnetosphere dynamo, Geophys. Res. Lett., 6, 577-600, 1979.

Kelley, M. C.: The Earth's Ionosphere. Academic Press, New York, 1989.

Lu, G., Lyons, L. R., Reiff, P. H., Denig, W. F., de la Beaujardiére, O., Kroehl, H. W., Newell, P. T., Rich, F. J., Opgenoorth, H., Persson, M. A. L., Ruohoniemi, J. M., Friis-Christensen, E., Tomlinson, L., Morris, R., Burns, G., and McEwin, A.: Characteristics of ionospheric convection and field-aligned current in the dayside cusp region, J. Geophys. Res., 100(A7), 11 845$11862,1995$.

Moretto, T., Vennerstrom, S., Olsen, N., Rastatter, L., and Raeder, J.: Using global magnetospheric models for simulation and interpretation of SWARM external field measurements, Earth Planets Space, 58, 439-449, 2006.

Ohtani, S., Zanetti, L. J., Potemra, T. A., Baker, K. B., Ruohoniemi, J. M., and Lui, A. T. Y.: Periodic longitudinal structure of fieldaligned currents in the dawn sector: large-scale meandering of an auroral electroject, Geophys. Res. Lett., 21, 1879-1882, 1994.

Ogino, T., Walker, R. J., and Ashour-Abdalla, M.: A magnetohydrodynamic simulation of the formation of magnetic flux tubes at the earth's dayside magnetopause, Geophys. Res. Lett., 16, 155-158, 1989.

Nenovski, P.: A signature of MHD surface wave field-aligned currents in plasmas, Physica Scripta, 53, 345-350, 1996.

Nenovski, P.: Surface waves and field-aligned currents in the magnetosphere, Adv. Space Res. 31, 1183-1193, 2003.

Nenovski, P., Danov, D., and Bochev, A.: On the field-aligned currents pattern formation in the magnetosphere, J. Atmos. Sol.Terr. Phy., 65, 1369-1383, 2003.

Nykyri, K., Otto, A., Lavraud, B., Mouikis, C., Kistler, L. M., Balogh, A., and Reme, H.: Cluster observations of reconnection 
due to the Kelvin_Helmholtz instability at the dawnside magnetosphere flank, Ann. Geophys., 24, 2619-2643, 2006, http://www.ann-geophys.net/24/2619/2006/.

Papitashvili, V. O., Belov, B. A., Faermark, D. S., Feldstein, Ya. I., Golyshev, S. A., Gromova, L. I., and Levitin, A. E.: Electric potential patterns in the Northern and Southern polar regions parameterized by the interplanetary magnetic field, J. Geophys. Res., 99, 13 251-13 262, 1994.

Papitashvili, V. O., Christiansen, F., and Neubert, T.: Field-aligned currents during IMF $\sim 0$, Geophys. Res. Lett., 28, 3055-3058, 2001.

Papitashvili, V. O. and Rich, F. J.: High-latitude ionospheric convection models derived from Defense Meteorological Satellite Program ion drift observations and parameterized by the interplanetary magnetic field strength and direction, J. Geophys. Res., 107(A8), 1198, doi:10.1029/2001JA000264, 2002.

Papitashvili, V. O., Christiansen, F., and Neubert, T.: A new model of FACs derived from high-precisionsatellite magnetic field data, Geophys. Res. Lett., 29(14), 1683, doi:10.1029/2001GL0114207, 2002.

Potemra, T. A.: Field-aligned (Birkeland) currents, Space Sci. Rev., 42(3+4), 295-311, 1985.

Raeder, J.: Global Magnetohydrodynamics - A tutorial, in: Space Plasma Simulations (Lecture Notes in Physics), edited by: Buechner, J., Dunn, C. T., and Scholer, M., Springer Verlag, Berlin, 615, 212-246, 2003.

Russell, C. T.: Geophysical coordinate transformations, Cosmic Electrodynamics, D. Reidel Publ. Company, Dordrecht-Holland, 2, 184-196, 1971.
Russell, C. T. and Elphic, R. C.: Initial ISEE magnetometer results: magnetopause observations, Space Sci. Rev., 22, p. 681, 1978.

Russell, C. T. and Elphic, R. C.: ISEE observations of flux transfer events at the dayside magnetopause, Geophys. Res. Lett., 6, 3336, 1979.

Stacievicz, K. and Potemra, T.: Multiscale current structures observed by Freja, J. Geophys Res., 103, p. 4315, 1998.

Stauning, P.: Field-aligned ionospheric current systems observed from Magsat and Oersted satellites during northward IMF, Geophys. Res. Lett., 29, 8005, doi:10.1029/2001GL013961, 2002.

Stauning, P.: Detection of currents in space by Oersted, SAC-C and CHAMP geomagnetic missions, in OIST-4 Proceedings, edited by: Stauning, P., Luhr, H., Ultre-Guerard, P., LaBresque, J., Purucker, M., Primdahl, F., Jorgensen, J., Christiansen, F., Hoeg, P., and Lauritsen, K., Copenhagen, Narayana Press, 121-130, 2003.

Weimer, D. R.: An improved model of ionospheric electric potentials including substorm perturbations and application to the Geospace Environment Modeling November 24, 1996, event, J. Geophys. Res., 106, 407-416, 2001a.

Weimer, D. R.: Maps of ionospheric field-aligned currents as a function of the interplanetary magnetic field derived from Dynamics Explorer 2 data, J. Geophys. Res., 106, 12 889-12 902, 2001b.

Zaneti, L. J., Potemra, T. A., Iijima, T., Baumjohann, W., and Bythrow, P. F.: Ionospheric and Birkeland current distributions for northward interplanetary field: Inferred polar convection, J. Geophys. Res., 89, 7453-7458, 1984. 